
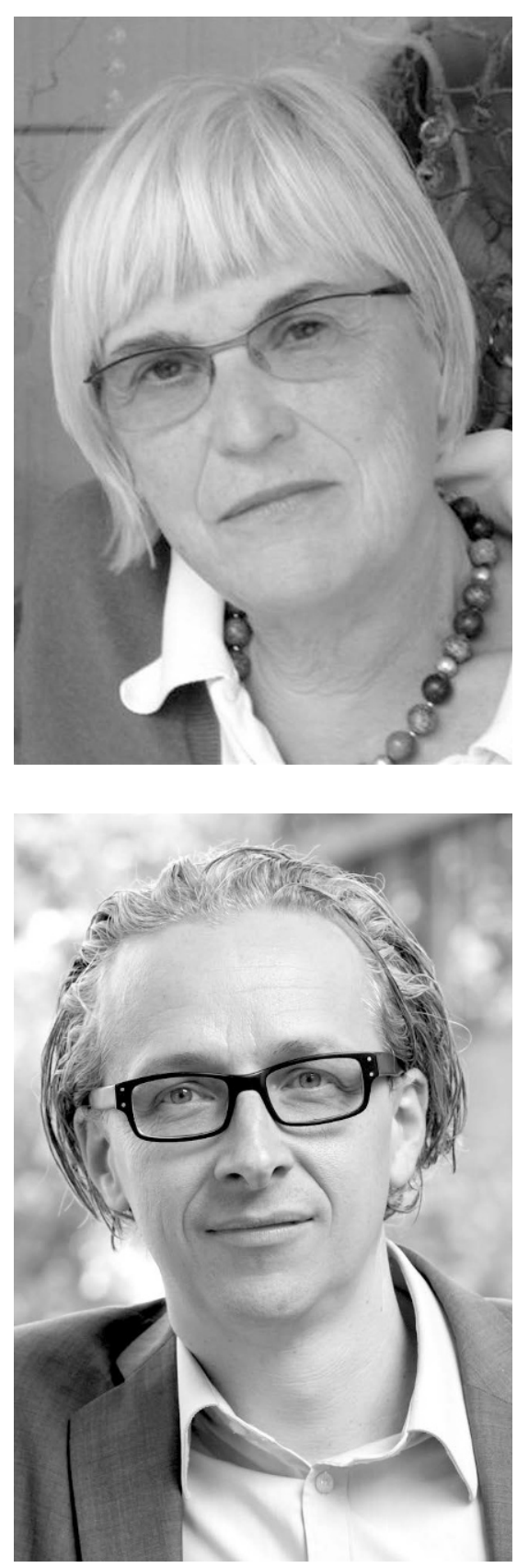

\section{Gundrechtliche Freiheiten in Störfällen}

Am 11. März 2020 ist einer der profiliertesten Kenner und Verteidiger der Menschenrechte, Burkhard Hirsch, gestorben. Sein Mitkämpfer und langjähriger Freund Gerhart Baum hat am 12. März 2020 einen persönlichen Nachruf geschrieben, der in dieser DuD veröffentlicht wird. Die Gedanken von Baum und Hirsch stärken das Bewusstsein für Grundrechtsgefährdungen. Dies betrifft ganz aktuell auch die Frage, wie mit den Grundrechtseingriffen zur Eindämmung der Corona-Krise auf Sicht umgegangen werden muss. Unsere Grundrechte bestimmen als oberste Grundsätze die gesamte Rechts- und Sozialordnung. Sie stehen im Dienst der Persönlichkeitsentfaltung und der Achtung der Menschenwürde. Grundrechtsbeschränkungen sind nur nach Maßgabe des Verhältnismäßigkeitsprinzips zulässig. Es trägt die „Hauptlast der Freiheitssicherung" (Dieter Grimm).

Welche Schutzmaßnahmen in Zeiten der Corona-Pandemie notwendig sind, lässt sich nur schwer beurteilen, da weder das wahre Ausmaß der Gefahren durch das Virus noch die Eignung und Erforderlichkeit der zu ergreifenden Maßnahmen sicher bestimmt werden können. Es muss aber alles getan werden, um die grundrechtsgefährdenden Entwicklungen der Bewegungs-, Berufs-, Versammlungs- und Religionsfreiheit $u$. a. genauer einzugrenzen und zu prüfen, ob es weniger einschneidende Maßnahmen gibt. Zu Recht betont der Europäische Datenschutzausschuss in seinen Leitlinien vom 21. April, dass solche Maßnahmen auf Dauer nicht hingenommen werden müssen; eine Befristung neuer gesetzlicher Kompetenzen und ihre unabhängige Evaluierung sind unerlässlich, um Geeignetheit und Erforderlichkeit der ergriffenen Maßnahmen auch für die Zukunft sachgerecht beurteilen zu können. ${ }^{1}$ Unter diesem Aspekt erging Ende März auch schon ein Appell der Europäischen Akademie für Datenschutz und Informationsfreiheit. ${ }^{2}$

Für den Schutz des Lebens und der körperlichen Unversehrtheit von Personen werden in Europa technische Überwachungsmöglichkeiten ins Spiel gebracht, die in Asien bereits im Einsatz sind. Es geht darum, präventive Maßnahmen auf der Technikebene einzusetzen, damit sich die Gefahrenherde gar nicht erst zu konkreten Gefahren oder gar Schäden auswachsen können. Die Frage ist u. a., ob Corona-Apps eingesetzt werden dürfen, um die Unterbindung oder Verzögerung von Neuansteckungen zu gewährleisten. Die damit verbundene Gefahr für die freie Kommunikation lässt sich nur bekämpfen, wenn Maßnahmen etwa durch den Einsatz einer CoronaTracing-App überhaupt tauglich sind und durch eine datenschutzfreundliche Technikgestaltung abgesichert werden können (Art. 25 DS-GVO - Datenschutz durch Systemgestaltung und Voreinstellungen). Wenn es allerdings darum gehen sollte, flächendeckend personenbezogene Bewegungsprofile zu erstellen und auszuwerten, dann ist die Grenze zulässiger Maßnahmen überschritten und das Grundrecht auf Privatheit und Datenschutz ausgehebelt.

Ganz grundsätzlich kann über Menschen- und Grundrechtsfragen nicht allein im nationalen Rahmen gesprochen werden. Deutschland ist Mitglied der EU und seine Verfassung lässt sich nicht mehr ohne die Einbindung in das Unionsrecht, insbesondere im Bereich der Grundrechte und der allgemeinen Rechtsgrundsätze, umreißen. Der Datenschutz ist ein herausragendes Anwendungsfeld des EU-Grundrechtsschutzes. Vor diesem Hintergrund befasst sich der Europarechtler Rudolf Streinz in diesem Heft mit dem Recht auf Vergessenwerden (Art. 17 DS-GVO) im Spannungsfeld von Unionsrecht und Verfassungsrecht. Er betrachtet anhand dieses Rechts beispielhaft das Datenschutzrecht als Ansatz für Neuerungen des Kooperationsverhältnisses von BVerfG und EuGH. Das Recht auf Vergessen ist dann auch Thema der Bei-

1 Guidelines 04/2020 on the use of location data and contact tracing tools in the context of the COVID-19 outbreak; https://edpb.europa.eu/sites/edpb/files/files/file1/edpb_guidelines_20200420_contact_tracing_ covid_with_annex_en.pdf.

2 https://www.eaid-berlin.de/appell-der-europaeischen-akademie-fuer-informationsfreiheit-und-datenschutzcorona-pandemie-bekaempfen. 
träge von Jörn Reinhardt und Maren Pollmann. Jörn Reinhardt wirft einen Blick auf unser Nachbarland Frankreich. Der Autor erläutert die Entscheidungen des französischen Staatsrats (Conseil d'État) zum Recht auf Vergessen und geht dabei insbesondere auf die unterschiedliche Rezeption der europarechtlichen Vorgaben durch Conseil d'État und BVerfG ein. Maren Pollmann wendet sich der Frage zu, wie weit nach den Maßgaben der EuGH-Rechtsprechung das Recht aufVergessenwerden auch länderübergreifend seine Geltung entfalten kann und ordnet diese Frage in einen völkerrechtlichen Kontext ein.

Schon seit jeher, gerade aber auch in Zeiten einer Pandemie, stellen sich Fragen der Sicherstellung von Freiheitsrechten als tragende Säulen im demokratischen Rechtsstaat. Unabdingbar ist, dass einer unabhängigen Institution die Aufgabe zukommt, personenbezogene Überwachungssysteme zu kontrollieren. Der Präsident des BayLDA Michael Will stellt die Frage, ob eine solche Datenschutzaufsicht künftig auch in Deutschland zentral geführt werden sollte und setzt sich in diesem Zusammenhang mit aktuellen Forderungen, insbesondere auch der Datenethikkommission, nach einer Ablösung der dezentralen föderalen Zuständigkeiten der Datenschutzaufsichtsbehörden der Länder auseinander.

Der Kommunikationswissenschaftler Tom Knieper und die Juristin Marie-Theres Tinnefeld befassen sich mit der Bedeutung politischer Satiren und Karikaturen für eine offene Demokratie. Sie begreifen Satiriker und Karikaturisten als Teil einer demokratischen Ordnung, die nicht im Lot ist. An zahlreichen Beispielen zeigen sie, wie Karikaturisten gerade mit ihren übertriebenen und verzerrten Darstellungen für den Meinungsbildungsprozess von zentraler Bedeutung sind, weil auf diese Weise auch aufgestaute Aggressionen und Ängste in schwierigen politischen Situationen freigelegt und öffentlich abgebaut werden können. Jedoch gefällt nicht allen betroffenen Personen in Politik und Gesellschaft dieser Ansatz. Zunehmend - und mit Erfolg - werden Presse und Medien auch von mächtigen Lobbyorganisationen wie der Anti Defamation League unter Druck gesetzt, Karikaturisten nicht mehr zu beschäftigen. Knieper und Tinnefeld befassen sich mit einzelnen Sachverhalten und untersuchen, inwieweit die scharfen Äußerungen mit Wort und Zeichenstift unter den Schutz der Meinungsfreiheit oder/und der Kunstfreiheit fallen sowie welche Grenzen ggfs. der Persönlichkeitsschutz allseitig setzt.

Marie-Theres Tinnefeld befasst sich in diesem Heft mit der umstrittenen Bedeutung von Informanten (Hinweisgebern) der Presse und den Regelungen zu ihrem Schutz. Skandale auf Regierungs- oder Wirtschaftsebene sind erst durch den Einsatz von Whistleblowern wie Snowden sowie investigativen Journalisten transparent geworden. Die Autorin geht in ihrem Beitrag auf die Rechtslage von Whistleblowern in Deutschland sowie auf jene Regeln der EU-Whistleblower-Richtlinie vom Oktober 2019 ein, die die Chancen von Informanten auf eine freie Meinungsäußerung und Autonomie jenseits von Repressalien erhöhen können.

Vier kürzere Beiträge runden das Heft ab: Maurice Oettel setzt sich mit Smart Humans und dem Schutz von Gedanken auseinander und unternimmt eine datenschutzrechtliche Einordnung von Brain-Computer Interfaces. Dennis Kipker stellt aus Anlass entsprechender Nachfragen sein Update zum EU Cybersecurity Act aus dem April-Heft der DuD nunmehr auch auf Englisch zur Verfügung. Merle Freye, Dennis Kipker, Ezekiel Rindstone und Doreen Mwamlangala untersuchen rechtsvergleichend die eHealth-Gesetzgebung hierzulande und in Tansania. In der Rubrik Best Practice schließlich ordnet Maximilian Schnebbe die Nutzung digitaler Assistenzsysteme in Industrie und Produktion datenschutzrechtlich ein.

\section{Marie-Theres Tinnefeld und Benedikt Buchner}

